\title{
La puntura ad occhiello su FAV nativa omero-cefalica superficializzata in paziente obesa
}

\section{F. Cavatorta}

Divisione di N efrologia e Dialisi, Ospedale di Imperia, Asl 1 imperiese

\section{Caso Clinico 3}

Nel giugno 2007 presso la Struttura Complessa di Nefrologia e Dialisi di Imperia è iniziato un programma di puntura delle fistole native con la tecnica ad occhiello. Dopo aver identificato il personale infermieristico idoneo, sono stati scelti inizialmente 4 pazienti, uno per turno dialitico, costituiti da un giovane paziente con FAV distale ben sviluppata ma particolarmente intollerante al dolore da puntura, un paziente con lesioni cutanee estese di natura psoriasica e con una FAV distale di recente confezionamento e di non facile pungibilità, un paziente con una FAV con numerosi aneurismi secondari a puntura cosiddetta ad "area" e un paziente con FAV prossimale.

Dopo 6 mesi, in considerazione dei risultati nettamente positivi, assenza di complicanze, relativa facilità nella manovra e notevole gradimento della stessa da par te del paziente, la tecnica è stata estesa ad altri 3 pazienti, raggiungendo un totale di 7 pazienti in trattamento con la puntura ad occhiello.

Si ripor ta il caso di una paziente facente par te del secondo gruppo di pazienti arruolati che è stata scelta per la difficoltà nel posizionamento degli aghi, in particolare di quello venoso.

Si tratta di una donna obesa di 64 anni rientrata in dialisi dopo il rigetto di un trapianto di rene durato oltre 15 anni durante i quali era stata sottoposta a procedura di angioplastica coronarica e a ovariectomia con chemioterapia per neoplasia ovarica sinistra, alla quale è stata confezionata una fistola prossimale omero-cefalica sinistra seguita dopo 2 mesi dalla superficializzazione del vaso cefalico, onde consentire una puntura più agevole.

Tuttavia, dopo alcuni mesi di funzionamento, a seguito del riscontro di una stenosi iuxta-anastomotica all'esame eco-
Doppler con riduzione della por tata dell'accesso valutata con il Transonic veniva effettuata una prima angioplastica con esito positivo.

Nei 2 anni successivi la FAV veniva nuovamente sottoposta a varie procedure di angioplastica e al posizionamento di due stent, uno in sede mediana del braccio e uno a livello dell'arco cefalico per lo sviluppo di una stenosi serrata recidivante.

$\mathrm{Nel}$ frattempo si intensificava il ritmo dialitico portandolo a 4 sedute settimanali per la tendenza a grave iperkaliemia e per ripetuti episodi di sovraccarico cardiocircolatorio nel periodo interdialitico lungo.

II posizionamento dei due stent contribuiva a ridur re la superficie utilizzabile per l'inserimento degli aghi, per cui la manovra di puntura dell'accesso con la tecnica "a scala di corda" diventava sempre più difficoltosa, appannaggio di pochi operatori particolarmente esperti e comunque spesso esposta a stravasi ematici; inoltre la distanza tra i due aghi era nella maggior parte dei casi di pochi centimetri (3-4 cm) con conseguente aumento del ricircolo per la difficoltà nel pungere a maggior distanza la vena cefalica dato il progressivo approfondimento del vaso venoso.

Si decideva pertanto, dopo aver informato la paziente di questa nuova tecnica e degli ottimi risultati ottenuti nel Centro a conferma di quanto riportato in letteratura, di iniziare la puntura ad occhiello prima con l'ago venoso e successivamente con l'ago arterioso selezionando 2 siti di inserzione a distanza di $7 \mathrm{~cm}$.

A tutt'oggi, dopo 4 mesi di utilizzo della tecnica, non si è verificata alcuna complicanza, a parte un tentativo infruttuoso con ago smusso nel sito venoso che ha richiesto la puntura con l'ago tagliente; la paziente oltre a non avvertire dolore alla puntura è molto più tranquilla e rilassata dato il minore rischio di errore nella manovra. 


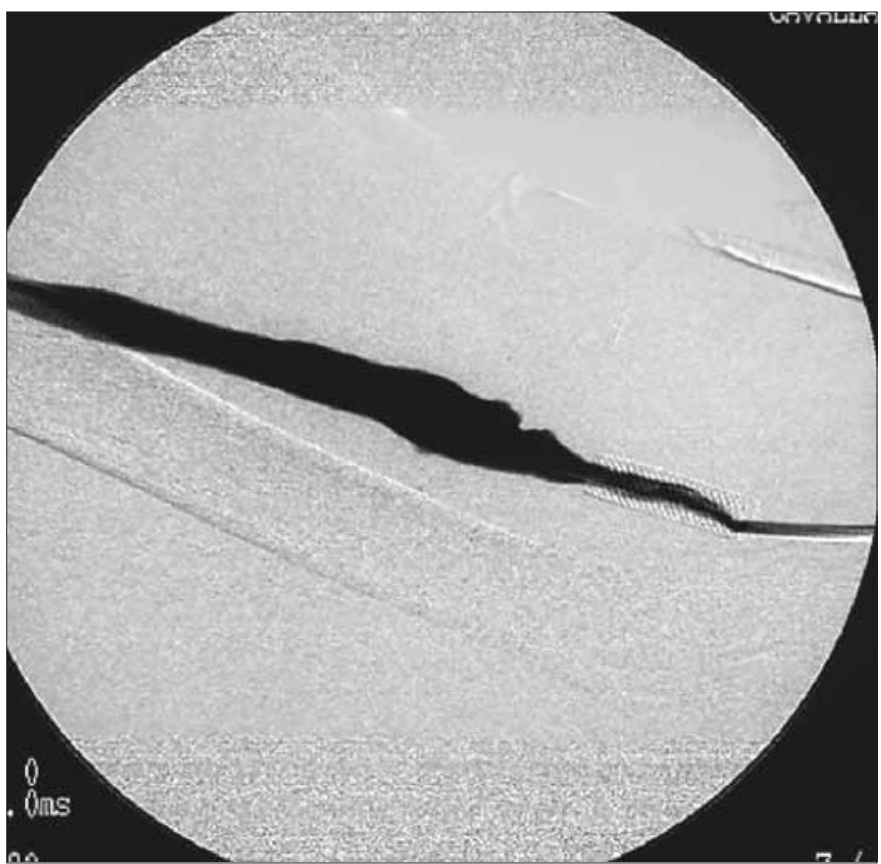

Fig. 1 - Stenosi intra-stent da iperplasia mio-intimale corretta con angioplastica.

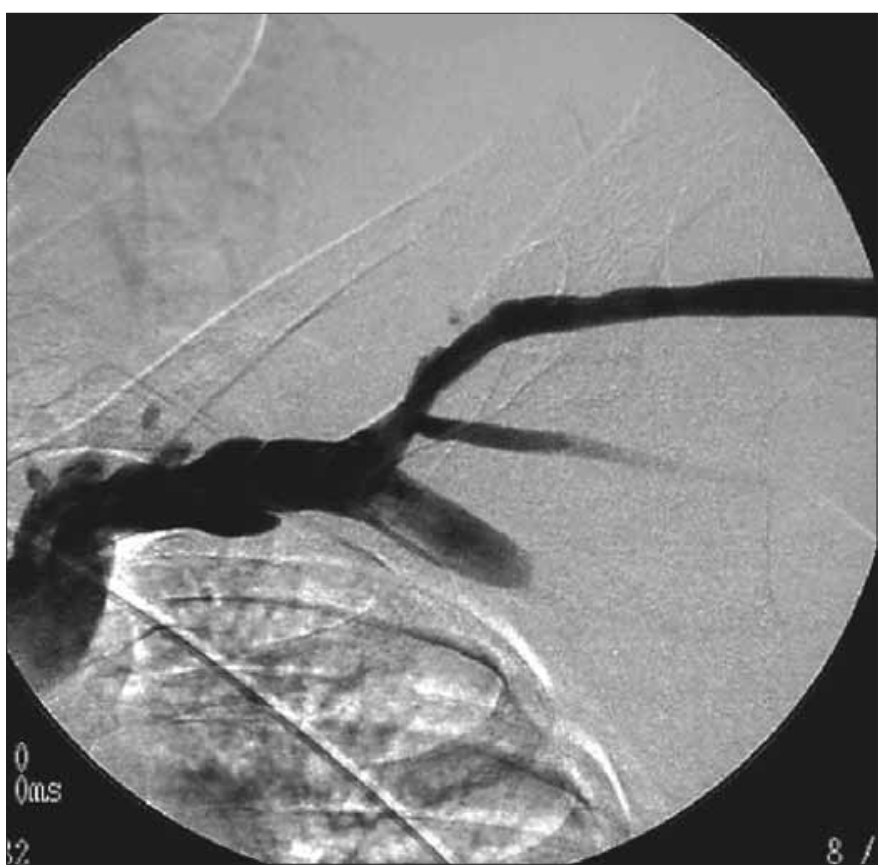

Fig. 3 - Correzione della stenosi dopo angioplastica.

Altri vantaggi osservati sono stati i tempi di emostasi molto più brevi e l'assenza di stillicidio attorno all'ago. Questo caso dimostra che la puntura ad occhiello, purché eseguita in modo corretto, è indicata nei casi di difficile aggressione dell'accesso con le altre consuete tecniche di puntura, in particolare nelle FAV con limitate aree di puntura, come in quelle con eventuali stent utilizzati per

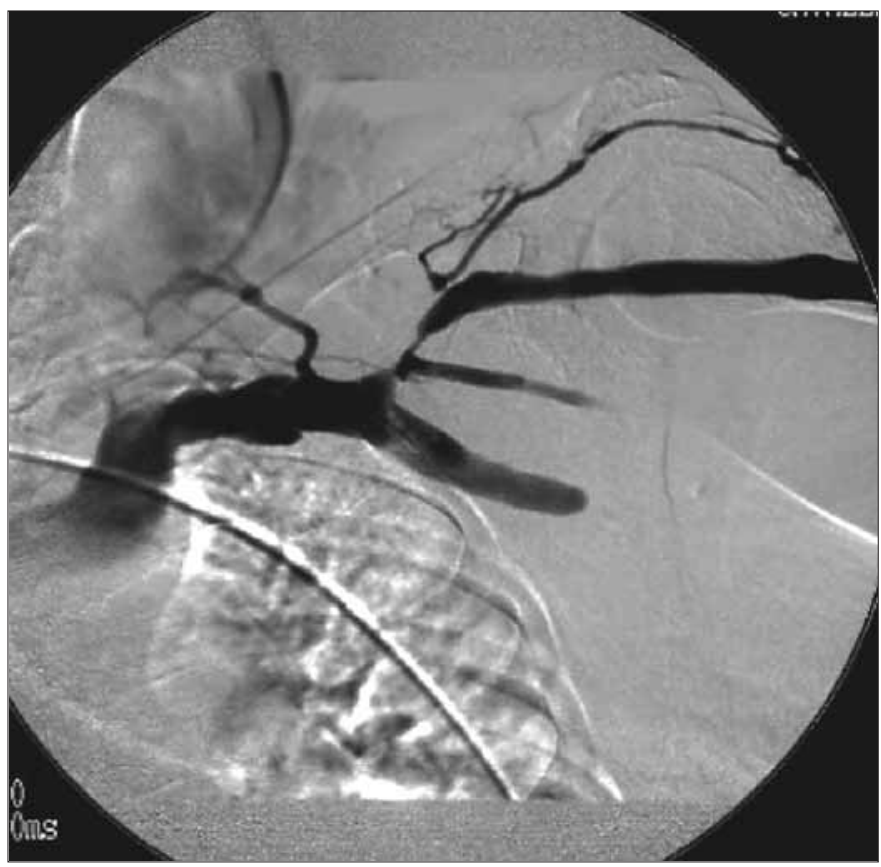

Fig. 2 - Stenosi serrata dell'arco cefalico.

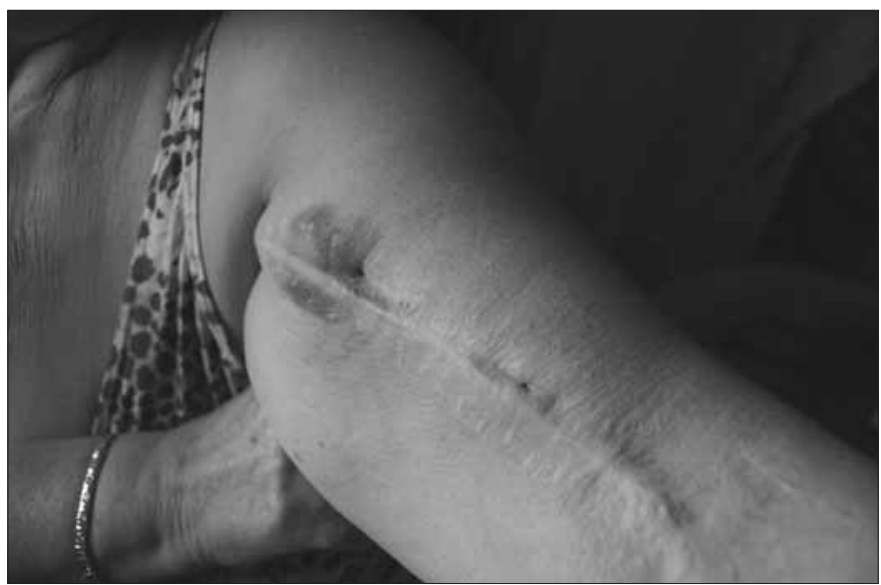

Fig. 4 - Foto del braccio.

la correzione di stenosi venose o con superficializzazione del vaso, superficializzazione che in genere non è mai molto estesa.

Si tratta di FAV destinate inevitabilmente allo viluppo di vistose dilatazioni aneurismatiche lungo il decorso del vaso con grave danno non solo estetico ma spesso anche funzionale dell'accesso.

Questa modalità di puntura della FAV pertanto, consentendo un accesso più sicuro con minori complicanze, dovrebbe garantire una maggiore sopravvivenza della fistola stessa.

email: fosco.cavatorta@libero.it 